\title{
Common Clinical Practice for Opioid-Induced Constipation: A Physician Survey
}

\author{
Flaminia Coluzzi $\mathbb{D}^{1,2}$ \\ Domenico Alvaro 3 \\ Augusto Tommaso Caraceni ${ }^{4}$ \\ Walter Gianni ${ }^{5}$ \\ Franco Marinangeli $\mathbb{D}^{6}$ \\ Giuseppe Massazza ${ }^{7}$ \\ Carmine Pinto ${ }^{8}$ \\ Giustino Varrassi $\mathbb{1 D}^{9}$ \\ Fabio Lugoboni ${ }^{10}$ \\ 'Department of Medical and Surgical \\ Sciences and Biotechnologies, Sapienza \\ University of Rome, Latina, LT, Italy; \\ ${ }^{2}$ Anesthesiology, Intensive Care, and Pain \\ Medicine Unit, Sant'Andrea University \\ Hospital, Rome, RM, Italy; ${ }^{3}$ Department of \\ Translational and Precision Medicine, \\ Gastroenterology Division, Sapienza \\ University of Rome, Rome, RM, Italy; \\ ${ }^{4}$ Palliative Care, Pain Therapy, and \\ Rehabilitation Unit, Fondazione IRCCS \\ Istituto Nazionale dei Tumori, Milan, MI, \\ Italy; ${ }^{5}$ Department of Internal Medicine and \\ Geriatric Medicine, University Hospital \\ Policlinico Umberto I, Rome, RM, Italy; \\ ${ }^{6}$ Department of Anesthesiology, Pain \\ Medicine, and Palliative care, University of \\ L'Aquila, L'Aquila, AQ, Italy; ${ }^{7}$ Division of \\ Physical Medicine and Rehabilitation, \\ Department of Surgical Sciences, University \\ of Turin and "Città della Salute e della \\ Scienza" University Hospital, Torino, TO, \\ Italy; ${ }^{8}$ Medical Oncology Unit, Clinical \\ Cancer Center, AUSL-IRCCS of Reggio \\ Emilia, Reggio Emilia, RE, Italy; ${ }^{9}$ Fondazione \\ Paolo Procacci, Rome, RM, Italy; \\ ${ }^{10}$ Department of Medicine, Addiction Unit, \\ University Hospital of Verona, Verona, VR, \\ Italy
}

Correspondence: Flaminia Coluzzi Department of Medical and Surgical Sciences and Biotechnologies, Sapienza University of Rome, Polo Pontino, Latina,

Corso della Repubblica 79, Latina, LT,

04100 , Italy

Tel +3906 33775673

Email flaminia.coluzzi@uniromal.it
Background: Opioid-induced constipation (OIC) remains an important clinical obstacle despite the availability of several guidelines and pharmacological options for its management. Here, we surveyed common practices and perceptions about OIC among physicians who prescribe opioids in Italy.

Methods: The online survey included 26 questions about OIC. Responses were analyzed descriptively and aggregated by physician specialty.

Results: A total of 501 physicians completed the survey. Most respondents (67\%) did not feel adequately educated about OIC despite general consensus regarding interest in the topic. Overall, $62-75 \%$ of physicians regularly evaluated intestinal function or OIC symptoms in patients receiving opioid therapy. The most common method for assessment was patient diary; few physicians used a validated instrument such as the Rome IV criteria. Psychiatrists and addiction specialists showed the lowest interest and poorest practices. Most respondents (78\%) preferred macrogol prophylaxis followed by macrogol plus another laxative for first-line treatment of OIC symptoms. Peripheral-acting mu opioid receptor antagonists (PAMORAs) were not widely used among physicians; $61 \%$ had never prescribed a PAMORA for OIC.

Conclusion: Our findings reveal important differences in clinical practice for OIC across physician specialties. Additional formative efforts are necessary to improve awareness about best practices in OIC.

Keywords: chronic pain, opioid, opioid-induced constipation, peripherally acting mu opioid receptor antagonist

\section{Introduction}

Opioid-induced constipation (OIC) is the most common type of opioid-induced bowel dysfunction, occurring in $51-87 \%$ of patients who take opioids for cancer pain, ${ }^{1-3} 41-57 \%$ of patients with chronic non-cancer pain, ${ }^{4}$ and an estimated $66 \%$ of patients receiving substitution therapy for opioid dependence. ${ }^{5}$ OIC symptoms are a common reason for interrupting opioid treatment and consistently diminish patient health-related quality of life (QoL). ${ }^{4,6}$ The effects of opioids on gastrointestinal functions are primarily mediated through opioid binding to mu and delta opioid receptors in the myenteric and submucosal plexus of the gastrointestinal tract. $^{7,8}$ Available treatment options include osmotic and stimulant laxatives, serotoninergic neuroenteric modulators (eg, prucalopride), and peripherally acting mu opioid receptor antagonists (PAMORAs), which are the unique agents that act directly on the mechanism of OIC.

Despite current knowledge about its prevalence and mechanism, OIC remains underdiagnosed and undertreated in a large proportion of patients. ${ }^{8}$ A nationwide 
study of more than 400 patients in France found that only $43 \%$ had been prescribed a medication for symptoms (most commonly an osmotic laxative) and treatment satisfaction was only moderate. ${ }^{9}$ Similarly, $50-80 \%$ of individuals taking a laxative for OIC report a lack of efficacy. ${ }^{10}$ A multinational survey in Europe found that $20 \%$ of patients were dissatisfied with their currently prescribed OIC treatment, ${ }^{8}$ suggesting that available treatments have limited efficacy or are otherwise not prescribed (or used) correctly by physicians and patients.

Deficits in OIC management and patient satisfaction are significantly related to the absence of a consensus definition for OIC across physician disciplines as well as the shortcomings of previous recommendations that were based on anecdotal evidence or expert opinion rather than the results of randomized clinical trials. ${ }^{11}$ In recent years, the availability of new clinical trials as a consequence of pharmaceutical development of inactive molecules for OIC has resulted in the publication of updated expert consensus in Europe ${ }^{12,13}$ and multi-disciplinary guidelines. ${ }^{2,14-17}$ Yet, evidence of low physician compliance with these guidelines suggests the existence of a gap between the current knowledge regarding OIC and realworld clinical practice. Here, we performed a nationwide physician survey assessing common clinical practices and perceptions about OIC in Italy in order to identify points of divergence with guideline recommendations as well as points for clarification, which require future efforts.

\section{Materials and Methods Study Design}

This study was a nationwide survey of physician perspectives and practices regarding OIC management in in Italy. Survey questions were elaborated by a multidisciplinary scientific board based on available literature, guidelines, and physician experience. The board was composed of 9 key-opinion-leaders (KOLs) in the field of oncology, orthopedic surgery and rehabilitative medicine, anesthesiology and pain medicine, gerontology, gastroenterology, palliative care, and psychiatry. The survey was active for a period of 6 weeks (February 18, 2020 to April 1, 2020) and communicated via e-mail using a password-protected web link to physicians of various specialties, involved in the management of patients under opioid therapy, including patients with cancer and non-cancer pain as well as those in opioid substitution therapy. Eligible physicians were identified from a private commercial database.
Survey responses were anonymized and handled via remote dispersed geographic participation. The study was not subject to approval by an ethical committee as per Italian law and international guidelines.

\section{Survey}

The survey included 2 filter questions designed to select physicians with sufficient exposure to patients in opioid therapy and 26 questions about common practices and perceptions related to OIC, including self-reported level of preparation and interest in OIC; diagnosis; prevention and treatment; perceived effects of OIC on patient QoL; and patient education (see Figure 1 for survey questions and Appendix 1 for the complete survey). Question responses were multiple-choice and either single response or multiple response depending on the nature of the question. Survey responses were tabulated by medical specialty or aggregated physician specialty category and summarized descriptively as the number and percentage.

\section{Results \\ Characteristics of Respondents}

The survey link was emailed to a total of 17,224 physicians in Italy; of these, 525 (3\%) accepted the invitation and were selected for inclusion in the survey. Twenty respondents were excluded during the initial screening questions and 61 provided only partial responses. Of the 501 respondents who were included after screening, nearly half were general practitioners (246 respondents, 49.1\%); $14.2 \%$ (71 respondents) were anesthesiologists/pain therapists; $7.2 \%$ (36 respondents) were oncologists; 5.6\% (28 respondents) were palliative care specialists; and 4.0\% (20 respondents) were psychiatrists (including specialists in addiction medicine). Other physician categories (orthopedic medicine, internal medicine, gastroenterology, gerontology, and rehabilitation medicine) had less than 20 respondents each.

\section{Awareness and Education on OIC}

Sixty percent of respondents indicated high interest in the topic of OIC (ie, score of 8-10 on a $0-10$ numerical rating scale); $36 \%$ indicated a medium level of interest (ie, score of 5-7); and 4\% indicated low or no interest (ie, score of 0-4) (Q18). When responses were categorized by physician specialty, anesthesiologists/pain therapists frequently expressed interest in OIC, whereas a majority of psychiatrists (70\%) expressed only 


\begin{tabular}{|c|c|}
\hline Number & Survey question \\
\hline Q1 & In clinical practice, do you see or treat patients who chronically use opioids? \\
\hline Q2 & In clinical practice, which molecules are used chronically by your patients? \\
\hline Q3 & What type of patients do you see in clinical practice who take chronic opioids? \\
\hline Q4 & In your clinical practice do you regularly evaluate pain? \\
\hline Q5 & At the center where you practice medicine, is intestinal function evaluated regularly? \\
\hline Q6 & In patients who chronically use opioids, do you systematically evaluate intestinal function? \\
\hline Q7 & What criteria do you use for constipation assessment? \\
\hline Q8 & $\begin{array}{l}\text { In clinical practice, in a patient receiving treatment with an opioid agonist, information about the possible emergence of constipation symptoms and indications for its } \\
\text { prevention/management }\end{array}$ \\
\hline Q9 & How many cases of opioid-induced constipation do you see in your clinical practice? \\
\hline Q10 & For the prevention of opioid-induced constipation, what strategies do you use in clinical practice? \\
\hline Q11 & Other than dietary and lifestyle measures, what first-line therapy do you find to be useful for the management of opioid-induced constipation? \\
\hline Q12 & In patients receiving laxative treatment for opioid-induced constipation, in what percentage do you prescribe a PAMORA (peripherally acting mu opioid receptor antagonist)? \\
\hline Q13 & In your opinion, to what degree can opioid-induced constipation influence the quality of life of your patients? \\
\hline Q14 & Do you agree that opioid-induced constipation can negatively influence adherence to analgesic therapy or opioid substitution therapy? \\
\hline Q15 & If you answered "yes" to question 14, please indicate the type of modifications that were applied to ongoing analgesic therapy. \\
\hline Q16 & $\begin{array}{l}\text { In your experience, what percentage of cases of opioid-induced constipation require modification of an opioid-containing therapeutic regimen for chronic pain or the dose of } \\
\text { methadone or buprenorphine in patients receiving substitution therapy for opioid dependence? }\end{array}$ \\
\hline Q17 & In clinical practice, do you find it useful to distinguish between opioid-induced constipation and constipation exacerbated by opioid use? \\
\hline Q18 & Express your interest in the topic of opioid-induced constipation on a scale from 0 (no interest) to 10 (maximum interest). \\
\hline Q19 & Do you feel adequately educated about the treatment of opioid-induced constipation? \\
\hline Q20 & In the management of candidates for surgical intervention, do you ask about pain symptoms and in particular if the patient has taken or is currently taking an opioid? \\
\hline Q21 & In the postoperative management of patients, do you systematically evaluate intestinal function? \\
\hline Q22 & What percentage of patients in your care continue to require an opioid analgesic after prosthetic surgical intervention through rehabilitation? \\
\hline Q23 & In postoperative rehabilitation, are patients who use opioids correctly informed about the possible emergence of constipation symptoms and indications for its management? \\
\hline Q24 & Do you believe that it would be useful to initiate awareness campaigns for opioid-induced constipation among patients receiving opioid therapy? \\
\hline Q25 & Do you evaluate exacerbating factors for constipation? \\
\hline Q26 & iswered "yes" to question 25 , which of the following factors do you evaluate? \\
\hline
\end{tabular}

Figure I Survey questions.

a moderate level of interest. Most respondents (67\%) also indicated that they did not feel adequately educated about OIC and were in favor of additional training, whereas $30 \%$ expressed satisfaction with their current level of education regarding OIC and 3\% were neither sufficiently informed nor interested in additional education regarding OIC (Figure 2) (Q19). When responses were further analyzed by aggregated physician specialty, the highest level of disinterest was expressed by psychiatrists $(15 \%$ not sufficiently informed and uninterested in further education), whereas most oncologists and palliative care specialists (66\% of respondents)

\section{Do you feel adequately educated about the treatment of opioid-induced constipation?}

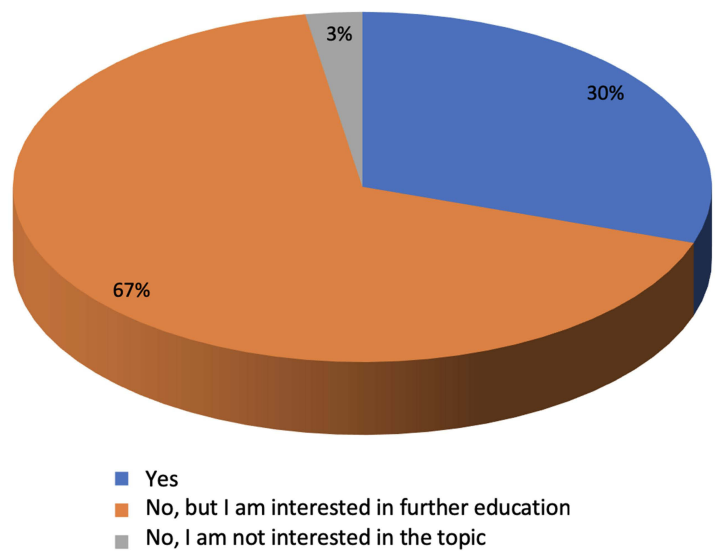

Figure 2 OIC education. expressed satisfaction with their current level of education.

Sixty-three percent of physicians indicated that they saw less than 5 cases of OIC per month in clinical practice (Q9). Among the aggregated physician categories, oncologists and palliative specialists saw the most cases of OIC (51.6\% ">5 per month", $21.0 \%$ ">20 per month"). Interestingly, $25 \%$ of psychiatrists indicated that they saw more than 5 cases per month despite a lack of reflected interest in OIC.

\section{Diagnosis of OIC}

In general, a majority of respondents $(63 \%)$ reported that OIC was evaluated at regular intervals or at every visit at their respective centers (Figure 3A) (Q5), and 62\% indicated that intestinal function was regularly evaluated in postoperative patients (Q21). Consistent with this result, $75 \%$ of respondents indicated that intestinal function was systematically evaluated in patients on long-term opioid therapy (Q6). Among the aggregated physician categories, $76 \%$ of oncologists and palliative care specialists evaluated intestinal function at every visit, while $50 \%$ of psychiatrists indicated that intestinal function was only evaluated if the patient or caregiver indicated a problem. Only $12 \%$ of respondents indicated that they used a specific scale or questionnaire to assess constipation. Among these, oncologists and palliative care specialists, together with the aggregated gastroenterologist/geriatric care/internal medicine group, were the categories that 


\section{A At the center where you practice medicine, is intestinal function evaluated regularly?}

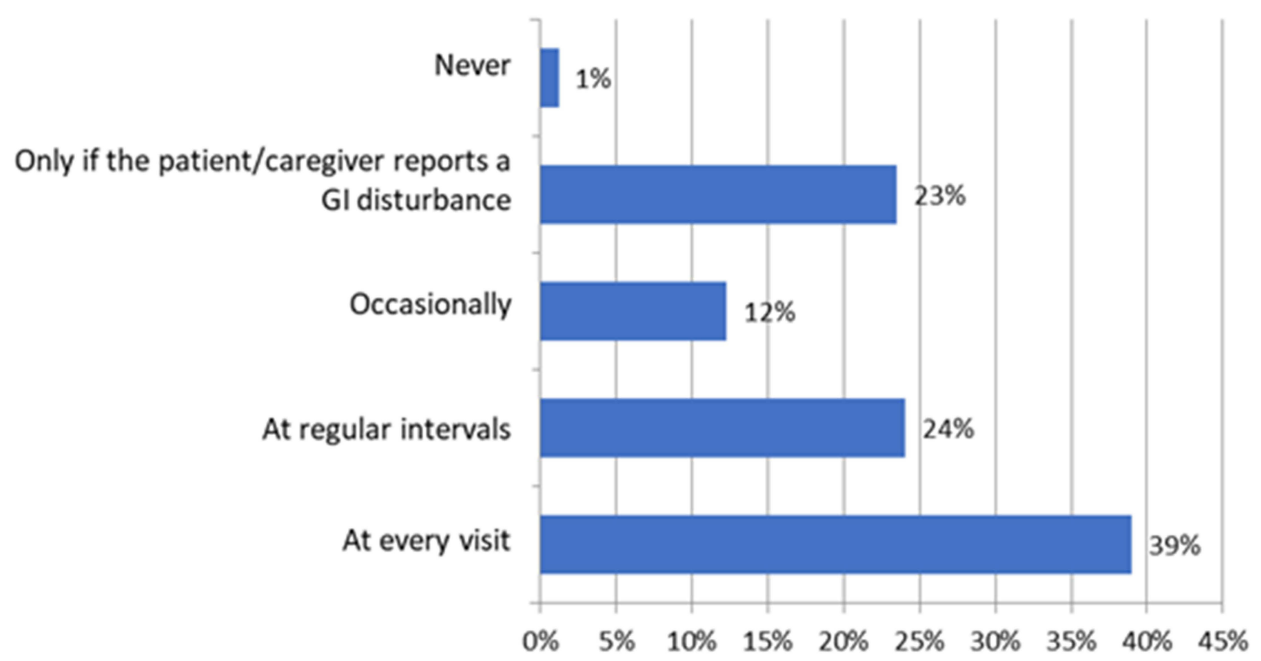

B What criteria do you use for constipation assessment?

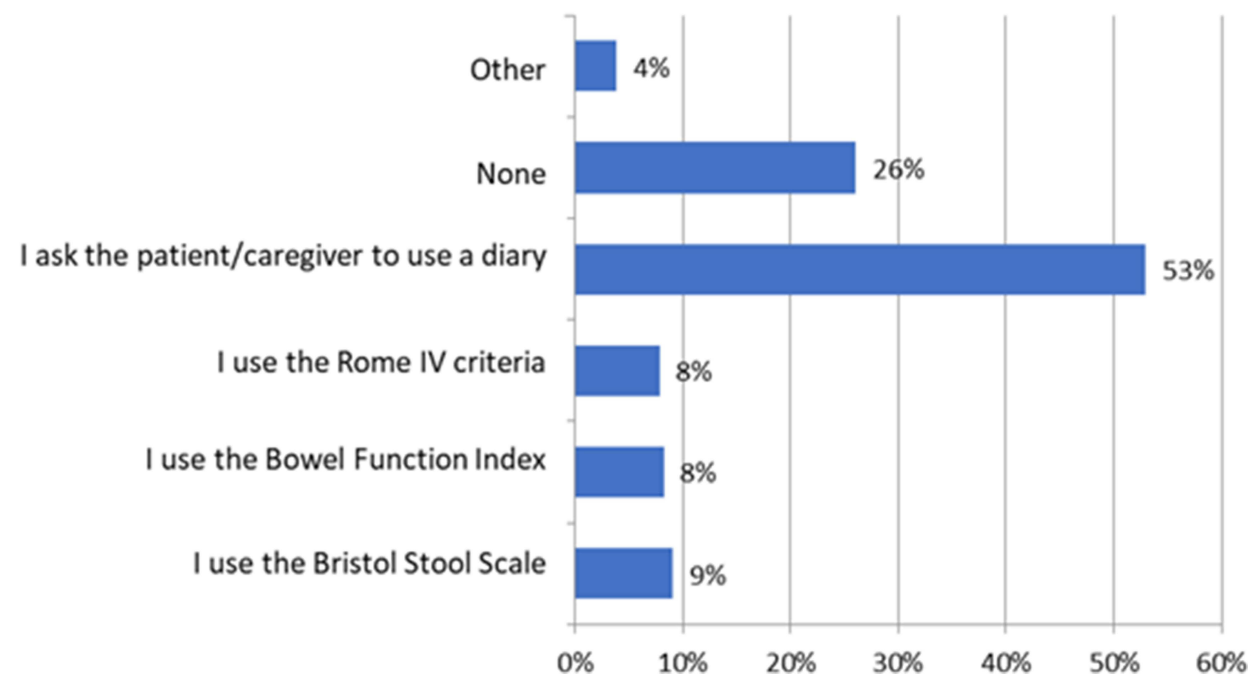

Figure $3 \mathrm{OIC}$ evaluation and assessment. (A) Evaluation of intestinal function. (B) Criteria for constipation assessment.

most frequently indicated a preference for the use of a specific scale or questionnaire for evaluating constipation ( $24 \%$ and $21 \%$, respectively).

Fifty-three percent of respondents indicated that OIC was most often diagnosed using patient diaries (Q7). Specific tools such as the Rome IV criteria, Bowel Function Index (BFI), and Bristol Stool Scale (BSS) were only used by $8-9 \%$ of respondents. When aggregated specialist categories were examined, oncologists and palliative care specialists generally favored the BFI (21\%) and BSS $(13 \%)$, while gastroenterologists favored the Rome IV criteria $(50 \%)$ and BSS $(50 \%)$. In contrast, $65 \%$ of psychiatrists reported that they did not use any method for evaluating constipation and the remainder rarely indicated the use of a specific instrument (Figure 3B).

Fifty-one percent of respondents agreed that it was always important to distinguish OIC from constipation exacerbated by opioid use, and an additional $32 \%$ of respondents expressed that this distinction was only important for the purpose of selecting appropriate treatment (Q17). To this end, almost all respondents (91\%) indicated that they evaluated exacerbating factors for constipation (Q25), which were subsequently specified as concurrent medications (35\%), comorbidities $(35 \%)$, and 
pain on defecation (20\%) (Q26). Concurrent medications tended to be more heavily considered by psychiatrists.

\section{OIC Prevention and Treatment}

Strategies for OIC prevention almost unanimously included advice regarding diet and exercise, including hydration (91\%), (Q10, multiple responses permitted), followed by laxatives $(57 \%)$ and evaluation of pharmacological interactions in patients receiving multiple therapies (28\%). Allowing the patient to self-medicate was rarely advised, although some anesthesiologists/pain therapists and addiction specialists favored this method. With regard to first-line treatment (excluding advice regarding diet, exercise, or hydration), $78 \%$ indicated a preference for macrogol as prophylaxis followed by a combination of macrogol plus another laxative (eg, senna, bisacodyl) for OIC symptoms. The second most common response was enema or glycerin $(10 \%)$, followed by senna or bisacodyl $(6 \%)$ and "other" (6\%; Figure 4A). In open comments, some explanations of "other" responses specified the use of peripherally acting mu-opioid receptor antagonists (PAMORAs; especially by anesthesiologists/pain therapists) and combinations of the listed therapies (eg, macrogol plus evacuating enemas as needed, recommended by palliative care specialists). Finally, $61 \%$ of respondents expressed that they never prescribed a PAMORA in patients with OIC receiving laxative treatment, whereas $21 \%$ indicated use of a PAMORA in up to $10 \%$ of cases (Figure 4B) (Q12). Oncologists were the most likely to use PAMORA $(70 \%)$, followed by

A Other than dietary and lifestyle measures, what first-line therapy do you find to be useful for the management of opioid-induced constipation?

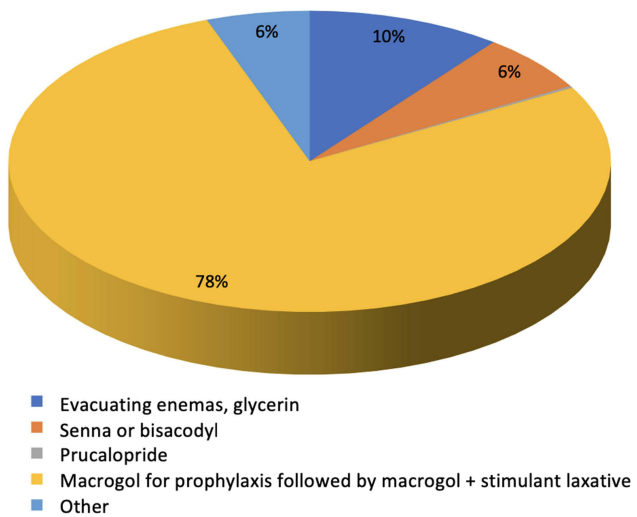

anesthesiologists/pain therapists (50\%). In contrast, $85 \%$ of psychiatrists indicated that they never used a PAMORA.

\section{QoL and Therapy Modification}

Ninety-nine percent of respondents agreed that OIC could potentially influence patient QoL to some extent, with $60 \%$ indicating a perceived significant influence on QoL (Figure 5A) (Q13). Moreover, 55\% of respondents acknowledged that OIC could negatively affect patient adherence to analgesic regimens or treatment for opioidrelated addiction (Figure 5B). When responses were stratified by aggregated specialty category, oncologists and palliative care specialists were more likely to acknowledge a negative effect of OIC on medication adherence, whereas $40 \%$ of psychiatrists indicated that the effect of OIC on QoL depended on the patient and 20\% did not believe it had any effect on QoL.

Among individuals who agree that opioid-induced constipation can negatively influence adherence to opioid therapy (Q14), 59\% indicated that changes to support medication adherence and address OIC symptoms would include the addition of an adjuvant therapy and a reduction in opioid dose, while $26 \%$ specified that they would not modify ongoing therapy and only $9 \%$ indicated a reduction in opioid dose only (Q15). Stratifying responses by aggregated specialty category revealed that psychiatrists broadly preferred to reduce the opioid dose only. In contrast, $52 \%$ of oncologists and palliative care specialists indicated that they would not modify ongoing therapy. Fifty percent of respondents indicated that modifying opioid therapy was

B In patients receiving laxative treatment for opioid-induced constipation, in what percentage do you prescribe a PAMORA?

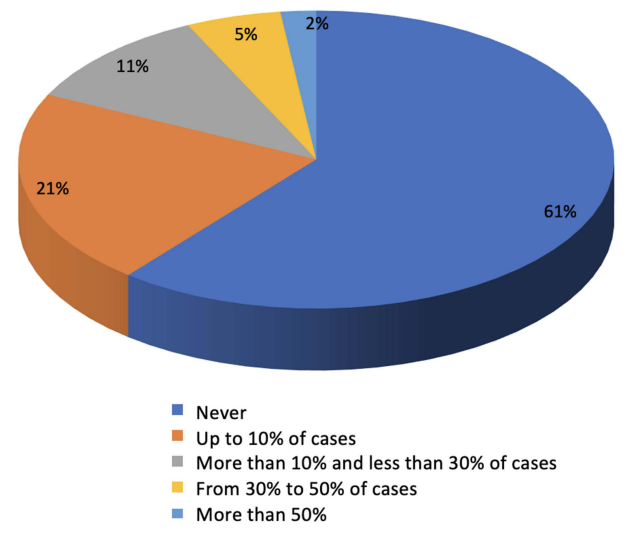

Figure 4 OIC management and treatment's strategies. (A) First line therapy for OIC. (B) PAMORA prescriptions. 
A In your opinion, to what degree can opioid-induce constipation influence the quality of life of your patients?

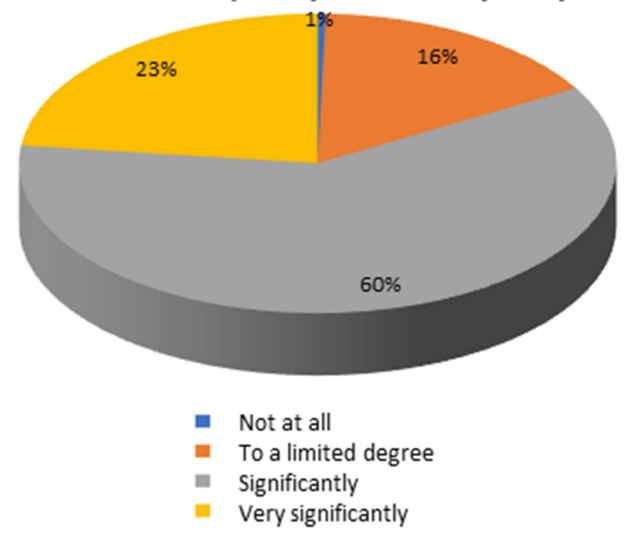

B Do you agree that opioid-induced constipation can negatively influence adherence to analgesic therapy or opioid substitution therapy?

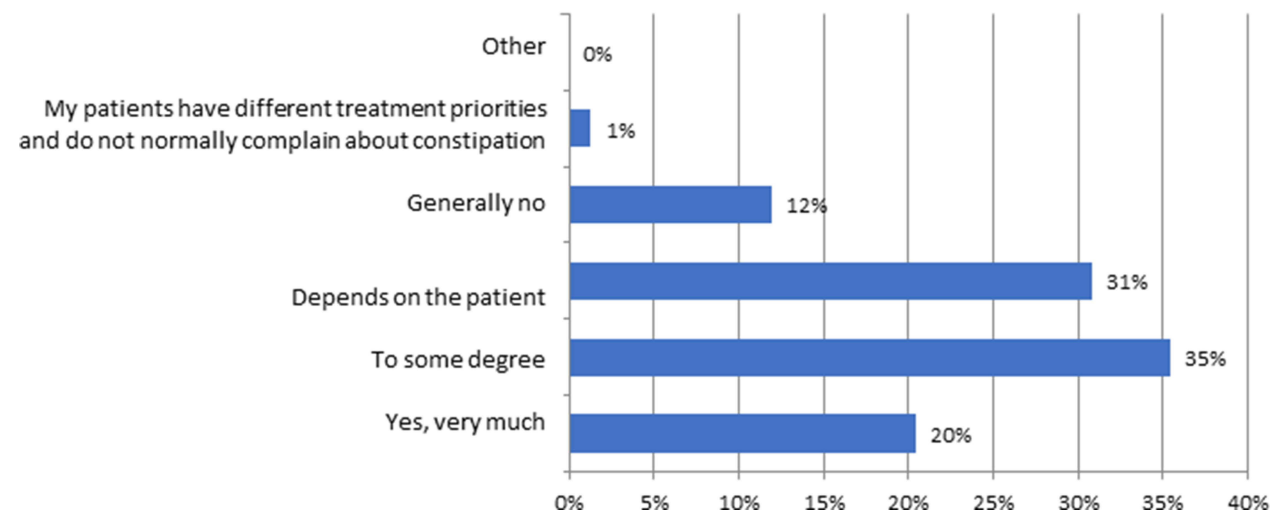

Figure $5 \mathrm{OIC}$ and impact on QoL. (A) OIC and QoL. (B) OIC and adherence to therapy.

only necessary to achieve a positive symptom outcome in $<10 \%$ of OIC cases (Q16).

\section{Patient Information}

Seventy-three percent of respondents indicated that patients were informed about the OIC and its prevention or eventual treatment prior to initiating opioid therapy, while $11 \%$ provided this information upon request and $10 \%$ only after the presentation of symptoms in order to avoid alarming the patient (Figure 6) (Q8). Yet, only 39\% of respondents indicated that similar information was provided to patients prior to administration of a postoperative opioid (Q23; in response to this question, 28\% indicated that they did not see this type of patient) and $17 \%$ provided a negative response. Nonetheless, there was nearunanimous agreement (96\%) in favor of awareness campaigns for OIC targeted to patients who receive long-term opioid therapy (Q24).

\section{Discussion}

OIC is one of the most common complications of opioid therapy and an obstacle to successful patient management. The present study characterized common clinical practice among physicians for OIC in Italy and revealed significant divergence across physician disciplines as well as mixed compliance with guideline recommendations.

The diagnosis of OIC has historically been complicated by the absence of a universally accepted definition, as the exact definition of OIC differs across medical specialties and clinical studies. ${ }^{18}$ While many physician respondents in our survey indicated a reliance on patient diaries (ie, self-report) for diagnosis of OIC, this measure is subject to patient as well as physician bias if a diagnosis is made without ensuring that specific criteria have been met. Diary and self-report measures are unreliable in patients with obsessive-compulsive disorders related to defecation, which are common among the elderly and patients with 


\section{In clinical practice, in a patient receiving treatment with an opioid agonist, information about the possible emergence of constipation symptoms and indications for its prevention/management}

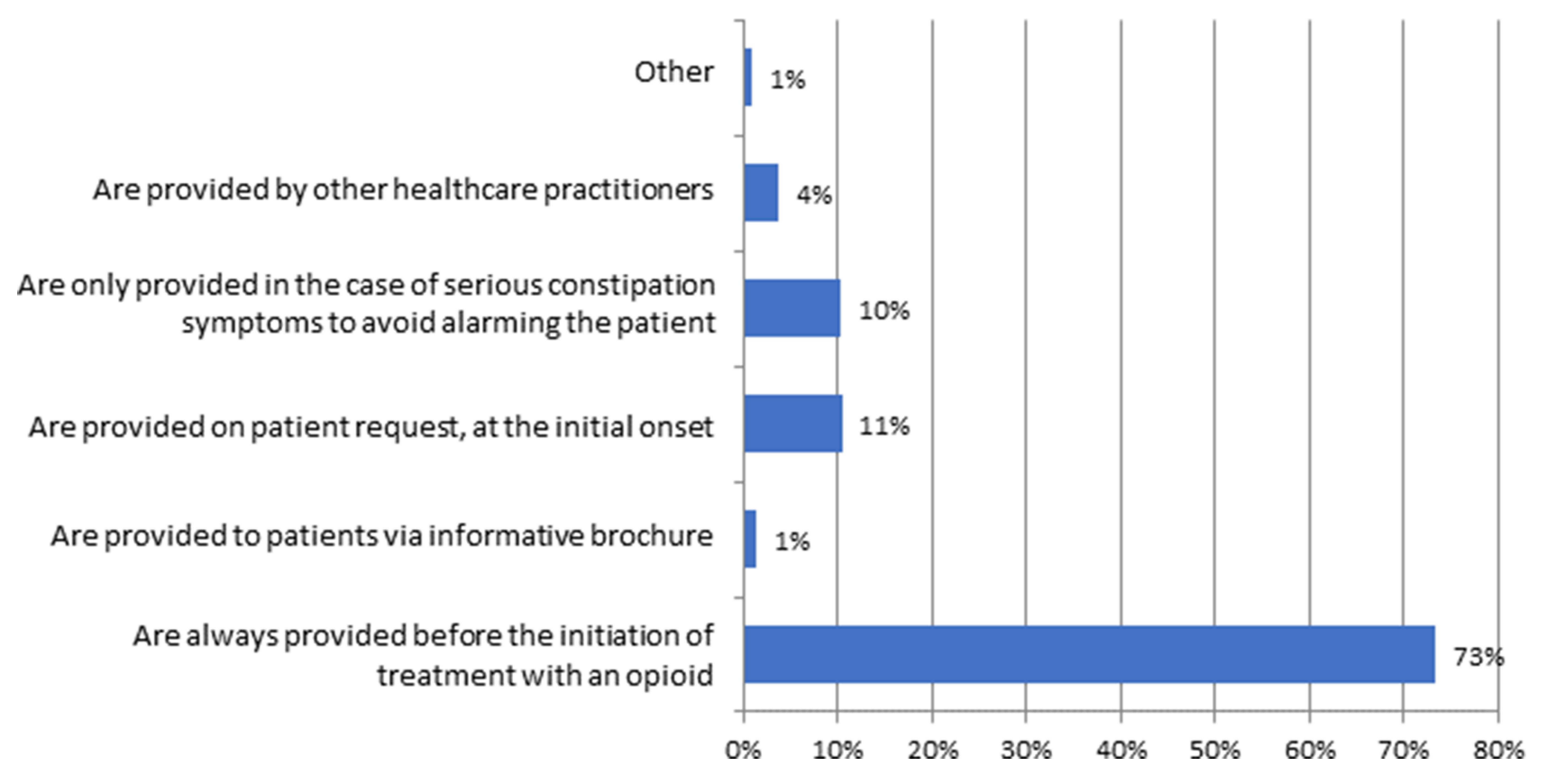

Figure 6 Patient information.

chronic constipation. ${ }^{19,20}$ In contrast, only $8-9 \%$ of respondents utilized a validated scale or instrument such as the Rome IV criteria, the BSS, and the BFI, and these respondents were typically oncologists or palliative care specialists and gastroenterologists. The Rome IV criteria represent an excellent tool for diagnosing OIC. ${ }^{12,21}$ Systematic use of this instrument could improve diagnostic accuracy and at least in part compensate for poor standardization of an OIC definition. Nonetheless, tools such as the BSS and BFI may be more appropriate for OIC symptom assessment and monitoring responses to treatment. In particular, the BSS is recommended as a quick and easy-to-use assessment for patients. ${ }^{7}$

In addition to regular screening for OIC symptoms, survey responses underscored a need to differentiate between OIC and constipation exacerbated by opioids in clinical settings, as the latter is potentially addressed through the modification of exacerbating factors rather than the opioid regimen itself. Opioids can exacerbate constipation associated with the use of other pharmacological agents such as antidepressants, antihistamines, antiepileptic agents, diuretics, and calcium antagonists. Patient comorbidities should also be considered, such as hypothyroidism or a history of chronic constipation or other gastrointestinal disturbance.

Guidelines for OIC have evolved in recent years alongside pharmaceutical development. The most recent recommendations for the pharmacological management of OIC identify standard osmotic and stimulant laxatives as firstline treatment, ${ }^{2,14-17,22}$ followed by PAMORAs for second-line treatment due to their peripherally restricted action and direct targeting of opioid receptors in the gastrointestinal tract. ${ }^{13,14}$ The survey results were congruent with guideline recommendations for first-line therapy, but few physicians reported ever using a PAMORA, with the exception of oncologists and to some extent anesthesiologists/pain therapists. It is noteworthy that PAMORA use in Italy was previously limited by the "nota 90," a regulation that only allowed their prescription through the national health system (NHS) for OIC in terminal patients, and the otherwise costprohibitive nature of these medications when prescribed outside of the NHS. Nota 90 was recently modified to allow the prescription of PAMORAs to all patients with 
OIC under the Italian NHS. Previous research has demonstrated that regulatory and reimbursement changes significantly influence the sale of medications in Italy, especially in the case of opioids, where such changes were associated with dramatic increases in opioid consumption during the period of 2000-2010. ${ }^{23}$ Accordingly, the recent modification of Nota 90 is expected to facilitate the prescription and consumption of PAMORAs as guidelinerecommended second-line therapy for OIC.

The PAMORAs naldemedine, naloxegol, and methylnaltrexone have all demonstrated good utility for the management of OIC, but are largely underutilized by clinicians. ${ }^{24}$ Methylnaltrexone is administered by subcutaneous injection and therefore has limited application for OIC given the invasive nature of treatment, ${ }^{25}$ whereas naloxegol and naldemedine are available in once-daily oral formulations and therefore have broader utility for the treatment of OIC. ${ }^{26,27}$ In the case of naldemedine, recent Phase III trials identified treatment-associated improvements in the number of bowel movements and QoL in patients in opioid therapy for cancer pain ${ }^{28,29}$ as well as chronic non-cancer pain. ${ }^{30}$ Another meta-analysis of available medications for OIC highlighted the usefulness of PAMORAs for managing OIC in patients with chronic non-cancer pain. ${ }^{31}$ After the failure of laxatives, PAMORAs provide an excellent alternative that does not compromise patient analgesia, but physicians may require additional education about the use of these agents before they fully enter into common clinical practice for OIC.

Contrary to research suggesting that the impact of OIC on QoL is often underappreciated by physicians, ${ }^{32}$ survey respondents appeared to be aware of the detrimental effects of OIC on patients. A common solution for addressing this problem was to modify existing opioid therapy with or without the addition of a laxative. In patients taking an opioid for pain, both OIC and underlying pain are factors that can significantly compromise patient QoL. ${ }^{4,33}$ Therefore, constipation symptoms and underlying pain should be equally considered when evaluating possible solutions to improve patient QoL, consistent with existing recommendations. ${ }^{13}$ Physicians should instead explore all available therapies, not limited to lifestyle changes and laxatives, to support all aspects of patient QoL.

An important theme emerging from the survey findings was the importance of training and education for both physicians and patients. Most physicians were interested in the topic of OIC and did not feel adequately educated about the topic, but were in favor of training or education. One important exception was in the case of psychiatrists (a category that included addiction specialists), who frequently expressed a level of disinterest or low engagement with the topic of OIC. This result was not surprising given a dearth of literature available on OIC in psychiatric or substance abuse patients, and may further reflect a disconnect in the patient-physician relationship in these care settings or a different perspective of physicians regarding the consequences of opioid use in patients with dependence. On the other hand, physicians were unanimously in favor of patient education campaigns. Previous literature has underscored "misalignments" between patients and clinicians regarding the adverse events of opioid therapy and their management. ${ }^{34}$ A previous multinational survey of 5 European countries reported that nearly $60 \%$ of healthcare professionals failed to adequately inform patients about constipation as a common side effect of opioid use. ${ }^{8}$ Adequate preparation and information sharing are critical for fostering positive patientprovider interactions (especially in a context of chronic pain management) and collaborative treatment decisionmaking. ${ }^{35}$ Accordingly, all physicians who prescribe opioids should have adequate education and training about OIC, regardless of specialty, and assume the responsibility of informing patients about and following the development of any OIC symptoms.

The present study had some limitations. The cohort of physician respondents included those with some inherent level of interest in OIC and was therefore targeted but small. Psychiatrists may have been underrepresented (20 respondents) while general practitioners were likely overrepresented (246 respondents) in our sample, although it may be argued that this ratio is generalizable to the actual proportions of these physicians in Italy. Interestingly, psychiatrists frequently expressed opinions that were at odds with those expressed by the overall cohort, suggesting that it may be necessary to direct future efforts towards this group of physicians in particular in order to prevent psychiatric patients and specifically those in treatment for opioid-related addiction or dependence from falling through the proverbial cracks of OIC management. ${ }^{36}$

\section{Conclusions}

The current state of OIC diagnosis and management in Italy remains inadequate, but is bolstered by apparent physician interest in improving the standard of care for patients in opioid therapy. All patients who receive a long- 
term opioid should be made aware of OIC as a possible adverse effect prior to initiating therapy and understand the available options for prophylaxis. Conversely, all physicians who treat patients in opioid therapy should be vigilant about monitoring for OIC symptoms using validated instruments and be well-educated about best practices, including options for pharmacological treatment.

\section{Ethical Statement}

This article does not contain any studies with human or animals, and it is not subject to approval by an ethical committee, according to the national law (Italian Law 11 January 2018 and Italian Legislative decree 14 May 2019, nr. 52). The participation in the survey was completely anonymous and voluntary, and all clinicians have indicated their willingness to voluntarily take part in the survey. A survey tool generates a numeric code for each participant which prevents the authors from identifying $\mathrm{him} / \mathrm{her}$ and results are reported in aggregated form. Survey responses were anonymized and handled via remote dispersed geographical participation. The invitation to the survey declared the content of the study and gave the opportunity to join to the project or deny the involvement, or terminate the participation at any time without penalty. By accepting our invitations each participant expresses the informed consent according to the legislation for the protection of privacy (Legislative Decree 196/2003 - European Regulation (EU) 679/2016).

\section{Institutional Review Board Statement}

Not applicable.

\section{Informed Consent Statement}

Not applicable.

\section{Acknowledgments}

The authors thank Claudia Laterza, MD of Sanitanova S.r.l and Ashley Symons, $\mathrm{PhD}$ for draft preparation and editorial assistance. All authors made a significant contribution to the work reported, whether that is in the conception, study design, execution, acquisition of data, analysis and interpretation, or in all these areas; took part in drafting, revising or critically reviewing the article; gave final approval of the version to be published; have agreed on the journal to which the article has been submitted; and agree to be accountable for all aspects of the work.

\section{Funding}

The research was funded by unconditional grants from Molteni Farmaceutici and Shionogi Inc.

\section{Disclosure}

FC is a speaker and consultant for Angelini, Grunenthal, Malesci, Molteni, Pfizer, and Shionogi. DA has received research grants and consultant fees from Intercept Pharma, Molteni, Shionogi, and Vesta, and is a consultant for Aboca. ATC has received research grants from Molteni, Gruenenthal GmbH, Prostrakan, Amgen, and Ipsen and is a consultant for Kyowa Kirin, Gruenenthal $\mathrm{GmbH}$, Pfizer, Helsinn Healthcare, Molteni, Shionogi, Italfarmaco, Sandoz International GmbH, Angelini Holding S.p.A., Mundipharma, and the Institute de Recherche "Pierre Fabre." WG has received research grants and other funding from Molteni and Shionogi. FM has received research grants and other funding from Molteni and Shionogi. GM has received research grants and other funding from Molteni and Shionogi. CP has received research grants and other funding from Molteni and Shionogi. GV is Member of the Advisor Boards of Abbott, Dompé, Malesci, Menarini International, Molteni, Mundipharma, Shionogi. Also, he is Member of the Speakers' Bureau of Berlin-Chemie, Dompé, MAP, Menarini International, MCAC, Molteni, Takeda. He has received funds for research by Dompé, Fondazione Maugeri and Pfizer. He is a Member of the Editorial Board of several scientific journals, and is Editor in Chief of Pain and Therapy. FL has received research grants and other funding from Molteni and Shionogi. The authors report no other conflicts of interest in this work.

\section{References}

1. Glare P, Walsh D, Sheehan D. The adverse effects of morphine: a prospective survey of common symptoms during repeated dosing for chronic cancer pain. Am J Hosp Palliat Care. 2006. 23 (3):229-235.

2. Drewes AM, Munkholm P, Simrén M, et al. Definition, diagnosis and treatment strategies for opioid-induced bowel dysfunction-Recommendations of the Nordic Working Group. Scand J Pain. 2016;11:111-122.

3. Tuteja AK, Biskupiak J, Stoddard GJ, Lipman AG. Opioid-induced bowel disorders and narcotic bowel syndrome in patients with chronic non-cancer pain. Neurogastroenterol Motil. 2010;22(4):e96.

4. Bell T, Annunziata K, Leslie JB. Opioid-induced constipation negatively impacts pain management, productivity, and health-related quality of life: findings from the National Health and Wellness Survey. J Opioid Manag. 5(3):137-144.

5. Troberg K, Håkansson A, Dahlman D. Self-rated physical health and unmet healthcare needs among swedish patients in opioid substitution treatment. J Addict. 2019;2019:7942145. 
6. Veiga DR, Mendonça L, Sampaio R, Lopes JC, Azevedo LF. Incidence and health related quality of life of opioid-induced constipation in chronic noncancer pain patients: a prospective multicentre cohort study. Pain Res Treat. 2018;2018:5704627.

7. Sizar O, Gea R, Gupta M. Opioid Induced Constipation. StatPearls; 2021. Available from: http://www.ncbi.nlm.nih.gov/pubmed/ 29630236. Accessed July 6, 2021.

8. Andresen V, Banerji V, Hall G, Lass A, Emmanuel AV. The patient burden of opioid-induced constipation: new insights from a large, multinational survey in five European countries. United Eur Gastroenterol J. 2018;6(8):1254-1266.

9. Ducrotté P, Milce J, Soufflet C, Fabry C. Prevalence and clinical features of opioid-induced constipation in the general population: a French study of 15,000 individuals. United Eur Gastroenterol J. 2017;5(4):588-600.

10. Cook SF, Lanza L, Zhou X, et al. Gastrointestinal side effects in chronic opioid users: results from a population-based survey. Alimentary Pharmacol Ther. 2008;27(12):1224-1232. doi:10.1111/ j.1365-2036.2008.03689.x

11. Brenner DM, Stern E, Cash BD. Opioid-related constipation in patients with non-cancer pain syndromes: a review of evidence-based therapies and justification for a change in nomenclature. Curr Gastroenterol Rep. 2017;19(3):12.

12. Alvaro D, Caraceni AT, Coluzzi F, et al. What to do and what not to do in the management of opioid-induced constipation: a choosing wisely report. Pain Ther. 2020;9(2):657-667.

13. Farmer AD, Drewes AM, Chiarioni G, et al. Pathophysiology and management of opioid-induced constipation: european expert consensus statement. United Eur Gastroenterol J. 2019;7(1):7-20.

14. Kampman K, Jarvis M. American Society of Addiction Medicine (ASAM) national practice guideline for the use of medications in the treatment of addiction involving opioid use. J Addict Med. 2015;9 (5):358-367.

15. Crockett S, Greer KB, Sultan S. (OIC) Guideline. Gastroenterology. 2019;156(1):228.

16. Crockett SD, Greer KB, Heidelbaugh JJ, et al. American gastroenterological association institute guideline on the medical management of opioid-induced constipation. Gastroenterology. 2019;156 (1):218-226.

17. Müller-Lissner S, Bassotti G, Coffin B, et al. Opioid-induced constipation and bowel dysfunction: a clinical guideline. Pain Med. 2017;18(10):1837-1863.

18. Gaertner J, Siemens W, Camilleri M, et al. Definitions and outcome measures of clinical trials regarding opioid-induced constipation: a systematic review. J Clin Gastroenterol. 2015;49(1):9-16.

19. Cosci F. "Bowel obsession syndrome" in a patient with chronic constipation. Gen Hosp Psychiatry. 2013;35(4):451.e1-3.

20. Towers AL, Burgio KL, Locher JL, Merkel IS, Safaeian M, Wald A. Constipation in the elderly: influence of dietary, psychological, and physiological factors. J Am Geriatr Soc. 1994;42(7):701-706.

21. Simren M, Palsson OS, Whitehead WE. Update on Rome IV criteria for colorectal disorders: implications for clinical practice. Curr Gastroenterol Rep. 2017;19(4):15.

Journal of Pain Research

\section{Publish your work in this journal}

The Journal of Pain Research is an international, peer reviewed, open access, online journal that welcomes laboratory and clinical findings in the fields of pain research and the prevention and management of pain. Original research, reviews, symposium reports, hypothesis formation and commentaries are all considered for publication. The manuscript

Submit your manuscript here: https://www.dovepress.com/journal-of-pain-research-journal
22. Caraceni A, Hanks G, Kaasa S, et al. Use of opioid analgesics in the treatment of cancer pain: evidence-based recommendations from the EAPC. Lancet Oncol. 2012;13(2):e58-68.

23. Musazzi UM, Rocco P, Brunelli C, Bisaglia L, Caraceni A, Minghetti P. Do laws impact opioids consumption? A breakpoint analysis based on Italian sales data. J Pain Res. 2018;11:1665-1672.

24. J V P, Christo PJ, LeQuang JA, Magnusson P. The Use of Peripheral $\mu$-Opioid Receptor Antagonists (PAMORA) in the management of opioid-induced constipation: an update on their efficacy and safety. Drug Des Devel Ther. 2020;14:1009-1025.

25. AIFA. RIASSUNTO DELLE CARATTERISTICHE DEL PRODOTTO Rizmoic. 2021. Available from: https://farmaci.agenzia farmaco.gov.it/aifa/servlet/PdfDownloadServlet?pdfFileName=foo ter_005017_047626_RCP.pdf\&retry $=0 \& s y s=m 0 b 113$. Accessed July 6, 2021.

26. AIFA. RIASSUNTO DELLE CARATTERISTICHE DEL PRODOTTO Moventig; 2019.

27. Coluzzi F, Scerpa MS, Naldemedine PJ, New A. Option for OIBD. J Pain Res. 2020;13:1209-1222.

28. Katakami N, Harada T, Murata T, et al. Randomized Phase III and extension studies of naldemedine in patients with opioid-induced constipation and cancer. J Clin Oncol. 2017;35(34):3859-3866.

29. Katakami N, Harada T, Murata T, et al. Randomized phase III and extension studies: efficacy and impacts on quality of life of naldemedine in subjects with opioid-induced constipation and cancer. Ann Oncol off J Eur Soc Med Oncol. 2018;29(6):1461-1467.

30. Saito Y, Yokota T, Arai M, Tada Y, Sumitani M. Naldemedine in Japanese patients with opioid-induced constipation and chronic noncancer pain: open-label Phase III studies. $J$ Pain Res. 2019;12:127-138.

31. Murphy JA, Sheridan EA. Evidence based review of pharmacotherapy for opioid-induced constipation in noncancer pain. Ann Pharmacother. 2018;52(4):370-379.

32. Cook S, Bell T, Sweeney C, Fehnel S, Hollis K. 883. J Pain. 2007;8 (4):S71.

33. Dueñas M, Ojeda B, Salazar A, Mico JA, Failde I. A review of chronic pain impact on patients, their social environment and the health care system. $J$ Pain Res. 2016;9:457-467.

34. Keller MS, Jusufagic A, Spiegel BMR. Patient and provider differences in the treatment of opioid-induced constipation: a qualitative study. BMC Gastroenterol. 2019;19(1):182.

35. Frantsve LME, Kerns RD. Patient-provider interactions in the management of chronic pain: current findings within the context of shared medical decision making. Pain Med. 2007;8(1):25-35.

36. Lugoboni F, Mirijello A, Zamboni L, et al. High prevalence of constipation and reduced quality of life in opioid-dependent patients treated with opioid substitution treatments. Expert Opin Pharmacother. 2016;17(16):2135-2141.

management system is completely online and includes a very quick and fair peer-review system, which is all easy to use. Visit http:// www.dovepress.com/testimonials.php to read real quotes from published authors. 